\title{
ARTICLES
}

\section{Refinement of the $P-T$ phase diagram of barium}

\author{
Markus Winzenick and Wilfried B. Holzapfel \\ Fachbereich Physik, Universität GH Paderborn, D-33095 Paderborn, Germany
}

(Received 6 February 1996; revised manuscript received 3 September 1996)

\begin{abstract}
The unusual $c / a$ ratio of the Ba-II/ "III" hcp phase was investigated as a function of pressure at different temperatures. The largest deviations from an ideal $c / a$ ratio for hcp was observed at room temperature just before the phase transition from the hcp to the complex Ba-IV phase. At higher temperatures this anomaly decreases and the $c / a$ ratio approaches the ideal value of hcp Ba- $\mathrm{V}$. This result together with the observation of a positive slope for the $T-P$ phase boundary from Ba-II/ 'III'" to Ba-IV support the view that Ba-IV should be considered only as a low temperature phase which separates the first hcp phase usually labeled Ba-II/“"III", at moderate temperatures from the seemingly different second hcp phase $\mathrm{Ba}-\mathrm{V}$ at higher pressures and ambient temperatures. [S0163-1829(97)04101-5]
\end{abstract}

\section{INTRODUCTION}

Although many earlier theoretical and experimental studies $^{1-5}$ contributed much to a better understanding of structures and phase boundaries of barium under pressure there remained some puzzling points. Barium and radium are the only alkaline earth metals which crystallize in the $c I 2$ structure at ambient conditions. At 5.5 GPa and room temperature barium shows its first transition to the $h P 2$ structure. ${ }^{6}$ This hexagonal structure is characterized by an unusual decrease of the $c / a$ ratio under pressure. ${ }^{4}$ In contrast to former differential thermal analysis and resistance measurements ${ }^{7-9}$ no further structural phase transition could be observed with x-ray diffraction up to $12 \mathrm{GPa}$ where barium transforms into the complex Ba-IV structure. ${ }^{1}$ Above $42 \mathrm{GPa}$ another phase with $h P 2$ structure, $\mathrm{Ba}-\mathrm{V}$, was repeatedly observed ${ }^{3,4,10}$ however, with a nearly normal $c / a$ ratio of 1.58(2). Theoretical calculations relate the structural transitions to $s \rightarrow d$-electron transfer or in other words to an increase in the $d$-band occupation under pressure. ${ }^{11}$ Linear muffin-tin orbital calculations reproduced the $c I 2 \rightarrow h P 2$ transition for barium, however, with a transition pressure of $10 \mathrm{GPa}^{12}$ The use of a "generalized pseudopotential", method $^{13}$ indicates "transition-metal character" for barium already at zero pressure. However, none of these calculations considered the complex Ba-IV phase and the upper $h P 2$ phase $\mathrm{Ba}-\mathrm{V}$. With the lack of any new experimental evidence for the II- "III" phase transition in the region of the initial $h P 2$ phase the anomalies in the melting curve between 6 and $9 \mathrm{GPa}$ present another puzzling phenomenon. Therefore, it appeared necessary to check all the phase boundaries I-II, II-“III,' and 'IIII' -IV also at elevated temperatures.

\section{EXPERIMENTAL DETAILS}

High pressures were generated with a diamond anvil cell $^{14,15}$ (DAC) with samples embedded in a $200 \mu \mathrm{m}$ diameter hole of an inconel gasket. White mineral oil was used as a pressure transmitting medium which protected also the sample against oxidation during the handling on air. Several ruby splinters of about $1 \mu \mathrm{m}$ diameter were inserted together with the sample into the gasket hole for pressure determination by the ruby luminescence technique ${ }^{16}$ using the nonlinear ruby scale. ${ }^{17}$ Temperatures were measured with a chromel-alumel-thermocouple attached to one of the diamonds. The accuracy of the temperature determination at the sample location is better than $\pm 4 \mathrm{~K}$ since possible temperature gradients were minimized by the use of a conventional external heating block surrounding the whole DAC. The thermal insulation of this system left only two small openings on the front and back sides for optical access and for transmission of the synchrotron radiation. The x-ray diffraction measurements were performed in the energy dispersive mode (EDXD) at HASYLAB (DESY, Hamburg). Details of the EDXD station at HASYLAB have been described previously. ${ }^{18,19}$

Estimates of pressures at elevated temperature were derived with reasonable accuracy from the measured lattice parameters by the use of these ambient temperature $p-V$ data taking into account the rather small thermal corrections evaluated in the appropriate high temperature limit of the Mie-Grüneisen approach ${ }^{20}$ with the form $\Delta P=3 \gamma k \Delta T / V$, where $V$ stands for the atomic volume, $\Delta T$ for the temperature difference with respect to ambient temperature, $k$ is the Boltzmann constant, and the thermodynamic Grüneisen parameter $\gamma$ was estimated with sufficient accuracy for each phase from the values for $K_{r}^{\prime}$ by the form ${ }^{21} \gamma=\left(K_{r}^{\prime} / 2\right)+\varepsilon$ with $\varepsilon=-0.5 \pm 0.4$. Since this thermal correction amounts at the highest temperatures of the present study just to 0.3 $\pm 0.1 \mathrm{GPa}$ this uncertainty does not reduce the following conclusions. Due to the phase mixing also at the phase transitions to the new $c F 4$ phase at elevated temperature and pressures, the equations of state (EOS) for the $c I 2$ and $h P 2$ phases could be used to put also close limits on the pressures where only this new phase was observed. Typically 


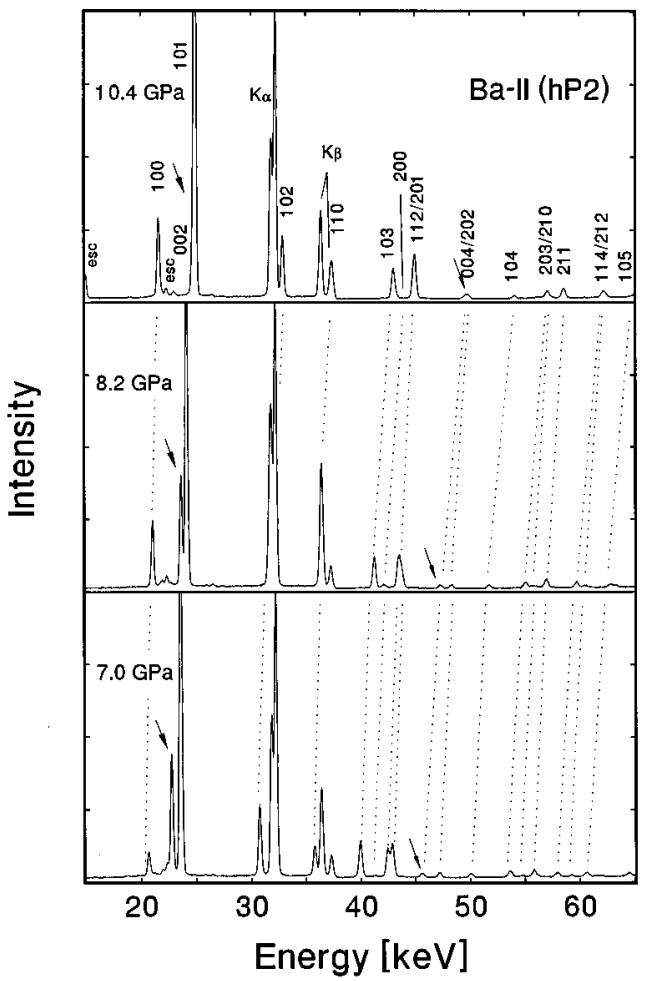

FIG. 1. EDXD pattern for $h P 2 \mathrm{Ba}-\mathrm{II}$ at ambient temperature and different pressures. Corresponding diffraction peaks are connected by dotted lines. The unusually strong shift of the (002) line and its higher order (004) is marked by arrows.

diffraction patterns were recorded in a few minutes and various sequences of measurements at almost constant temperature but increasing (or decreasing) pressure were performed to determine the phase transitions and the variation in the $c / a$ ratio for the $h P 2$ phases also at higher temperatures.

A detailed discussion of the EOS data from the present study will be given elsewhere. ${ }^{22}$ However, for completeness the parameters $V_{r}, K_{r}$, and $K_{r}^{\prime}$ for the volume, the bulk modulus, and its pressure derivative were determined for the $c I 2$ (Ba-I) and the first $h P 2$ phase (Ba-II/“'III') from this study $^{22}$ together with previous literature data ${ }^{1,4,23,24}$ by least square fits with a second order Birch equation. ${ }^{25}$ The parameters are $V_{0}=0.06372 \mathrm{~nm}^{3}, K_{0}=8.93 \mathrm{GPa}^{24} K_{0}^{\prime}=2.8(5)$ using $P_{r}=0 \mathrm{GPa}$ for the $c I 2$ phase, and $V_{r}=0.043(2) \mathrm{nm}^{3}$, $K_{r}=14(8) \mathrm{GPa}$, and $K_{r}^{\prime}=3(2)$ with $P_{r}=5.5 \mathrm{GPa}$ for the first $h P 2$ phase.

\section{RESULTS AND DISCUSSION}

Typical patterns for $h P 2$ Ba-II are shown in Fig. 1 for 7.0, 8.2, and 10.4 GPa. To reduce the texture, the patterns at 8.2 and $10.4 \mathrm{GPa}$ were recorded with a sample spinner. ${ }^{26}$ The comparison of these patterns with the one for 7.0 GPa shows that the smaller intensity of the (100) line in this last case results just from texture. The large decrease of the $c / a$ ratio within the $h P 2$ structure of Ba-II is illustrated by the unusually strong shifts of the (002) and (004) line in the spectra which leads to a merging of these two lines with the (101) and (202) lines, respectively, at pressures above 10.4 GPa. However, no discontinuous or unusual changes in the

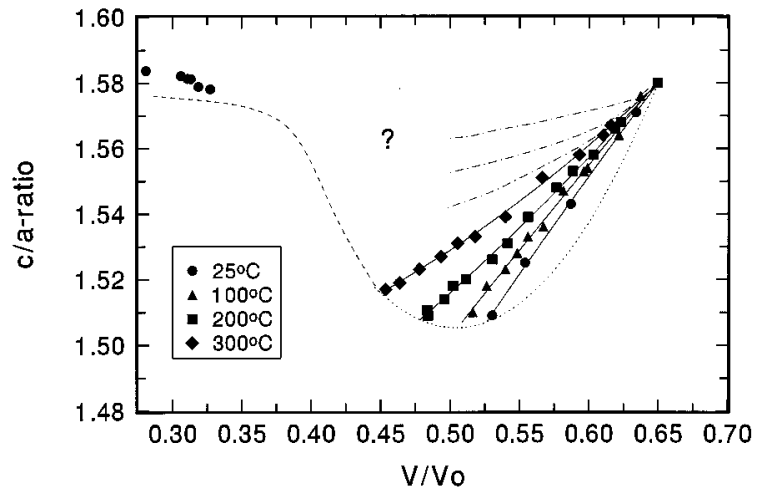

FIG. 2. $c / a$ ratio of $h P 2 \mathrm{Ba}-\mathrm{II}$ and $h P 2 \mathrm{Ba}-\mathrm{V}$ at different pressures and temperatures. Dot-dash lines represent extrapolations for 400,500 , and $600{ }^{\circ} \mathrm{C}$. Values of the $c / a$ ratio just before the phase transition to $\mathrm{Ba}-\mathrm{IV}$ are given by the dotted curve, which represents therefore the limit of the stability range for the $h P 2$ structure. A possible extrapolation of this curve to the data of $\mathrm{Ba}-\mathrm{V}$ is given by the dashed curve.

line positions or intensities are noticed in the pressure range around $8 \mathrm{GPa}$ where earlier measurements ${ }^{7,8,27}$ had given some indications for a phase transition to a new phase. This new phase is labeled here now Ba- "III" since this transition is not confirmed by the present study in close agreement with some previous measurements. ${ }^{1,4}$ These authors also could not find any sign of a phase transition in this pressure range. One possible explanation for the spurious "observation" for a phase transition from Ba-II to "III" can be related to the observation of a phase transition in $\mathrm{BaO}$ (Ref. 28) around 10 $\mathrm{GPa}$, which was also observed in some experiments of the present series when oxidation occurred during the handling of the sample.

Therefore it can be noted that all spectra for barium in the temperature range up to $300{ }^{\circ} \mathrm{C}$ and pressures between 7 and $12 \mathrm{GPa}$ are perfectly represented by just one $h P 2$ phase with only continuous changes in $c / a$ as illustrated in Fig. 2 where the data are plotted with respect to the directly measured volume to avoid additional uncertainties from pressure estimates. Most strikingly, Fig. 2 shows a strong temperature dependence of $c / a$ at constant volume for $V / V_{0}$ around 0.5 and the extrapolation of this temperature dependence to higher temperatures indicated in Fig. 2 by three additional isotherms for 400,500 , and $600{ }^{\circ} \mathrm{C}$, respectively, leads to the suspicion that the anomalous decrease in $c / a$ with increasing pressure (or decreasing volume) may disappear completely at elevated temperatures.

Furthermore, Fig. 2 also illustrates that the low pressure $h P 2$ phase, now labeled Ba-II for the whole region II + "III," extends to higher pressures (or smaller volumes) at elevated temperatures. In this figure the dotted line represents the borderline of the stability region of $h P 2$ below 300 ${ }^{\circ} \mathrm{C}$ according to the present results. The dashed line extrapolates this line just qualitatively to higher temperatures (and pressures) to point out that for higher pressures a continuous increase of $c / a$ along this line towards the more regular values for the $h P 2$ phase $(\mathrm{Ba}-\mathrm{V})$ would not contradict any physical rules. In any case, this region deserves further experimental investigations and the present study can only give hints for some likely behavior in this range. Obviously, the 


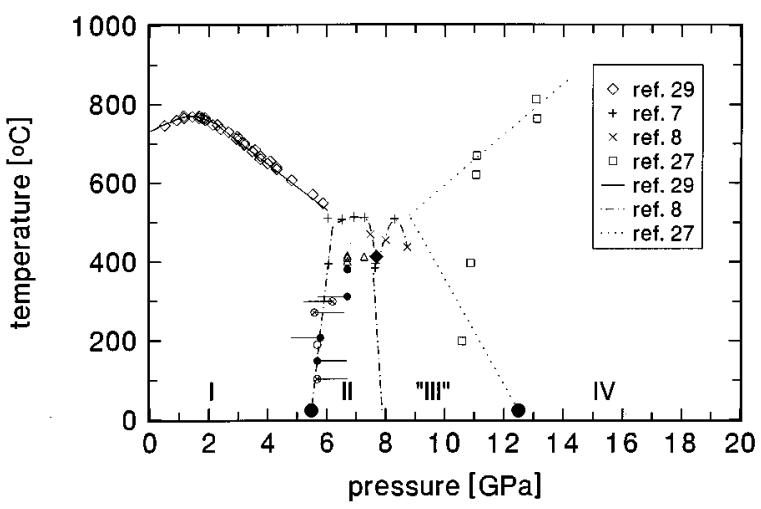

FIG. 3. Phase diagram for barium according to literature data together with present data for the I-II phase boundary, for the new $c F 4$ phase $\Delta$, as well as for melting $\bullet$. Small open and solid circles represent conditions where pure $c I 2$ and $h P 2$ spectra were observed, respectively. In the same way ranges of phase mixture are represented by crossed circles. Tags at the left or right side of these circles mark data points taken after increasing or decreasing the pressure. Large solid circles represent the phase transitions at ambient temperature.

present observation of an extended region of stability for $h P 2 \mathrm{BaII} /$ "III' towards higher pressures at higher temperatures is in conflict with the conventional phase diagram of barium shown in Fig. 3, where the negative slope of the II/ "III' -IV boundary resulted from a tentative interpolation $^{27}$ from ambient temperature to the apparent minimum in the melting curve. ${ }^{8}$ This interpolation was based on small discontinuities in the isobaric resistivity measurements, illustrated by the open squares in Fig. 3. It should be noted that this interpolation does not perfectly meet these resistivity anomalies casting some doubts on the significance of these resistivity data.

Furthermore, one should notice that the temperature scale in the later of the two publications from the same group ${ }^{7,8}$ was erroneously shifted up by $100{ }^{\circ} \mathrm{C}$ and this mistake has not been noticed in recent publications. ${ }^{4,27}$ The necessary correction is taken into account in Fig. 3 with the consequence that melting data above $10 \mathrm{GPa}$ do not reproduce the third dip in the melting curve near $9 \mathrm{GPa}$. Obviously this third dip near $9 \mathrm{GPa}$ is supported only by one data point from these publications, ${ }^{7,8}$ and one may have the suspicion that the temperature values of the second series ${ }^{8}$ just deviate from the earlier series ${ }^{7}$ of the same group. This means that the experimental evidence for the third triple point appears to be very weak. Additional data on the I-II phase boundary are shown in Fig. 3. These data were obtained by heating at almost constant pressure along the I/II phase line. Due to small decrease of the pressure on heating at constant load, the first data point at $103{ }^{\circ} \mathrm{C}$ (crossed circle) represents the first appearance of $\mathrm{Ba}-\mathrm{I}$ lines in the earlier Ba-II sample. On marginal pressure increase, the sample was completely transformed into $\mathrm{Ba}-\mathrm{II}$ and maintained in this structure upon heating up to $150{ }^{\circ} \mathrm{C}$ as illustrated by the next data point (full circle). Further heating resulted in back transformation to $\mathrm{Ba}-\mathrm{I}$ (open circle at $190^{\circ} \mathrm{C}$ ) and further marginal pressure increase completed with almost no hysteresis the transformation to Ba-II (solid circle at $208^{\circ} \mathrm{C}$ ). In the same way the next data points at higher temperatures denote either phase

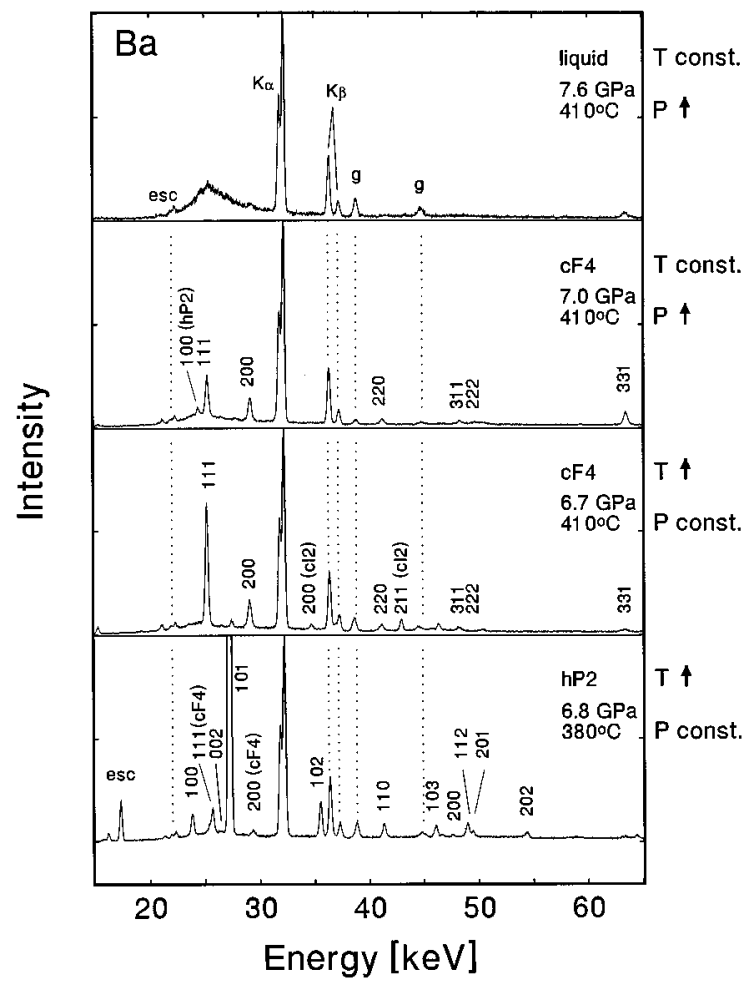

FIG. 4. Typical spectra for the phase transitions $h P 2 \rightarrow c F 4 \rightarrow$ liquid for barium. Escape, fluorescence, and gasket peaks are connected by dotted lines. The arrows on the right side indicate the preceeding $P-T$ change for each pattern.

mixture (crossed circles) along the Ba-I/II phase line or pure Ba-II spectra indicated by solid circles.

Thus, up to $300{ }^{\circ} \mathrm{C}$ the present data confirm the previous data for this phase line. However, above $380{ }^{\circ} \mathrm{C}$ at $6.8 \mathrm{GPa}$ the diffraction spectra show definitely a phase transition to a new $c F 4$ structure. Diffraction spectra for this new $c F 4$ phase are given in Fig. 4 with a sequence for increasing temperature. Increasing pressure at $410{ }^{\circ} \mathrm{C}$ to $9 \mathrm{GPa}$ produces first pure $c F 4$ spectra and then gives indications for the dip in the melting curve near $7.7 \mathrm{GPa}$ by complete loss of the diffraction peaks but with constant intensity of the fluorescence lines and an increase of a diffuse "background" typical for amorphous or liquid samples. The atomic volume for the new $c F 4$-Ba-III phase fits to the neighboring $c I 2-\mathrm{Ba}-\mathrm{I}$ and $h \mathrm{P} 2$-Ba-II phases. The total volume decrease $\Delta V_{\mathrm{I} \rightarrow \mathrm{II}}$ of $7.7 \%$ splits up into $\Delta V_{\mathrm{I} \rightarrow \mathrm{III}} \approx 3.3 \%$ and $\Delta V_{\mathrm{III} \rightarrow \mathrm{II}} \approx 4.4 \%$. Considering the fact that also $\mathrm{BaO}$ at $7 \mathrm{GPa}$ and ambient temperature crystallizes in a cubic structure it was carefully checked that neither at the beginning nor at the end of the measurement any oxide was present in this experiment and also the lattice parameters would not fit to the literature data for $\mathrm{BaO}$ at $7 \mathrm{GPa}^{28}$ Therefore a formation of oxide under this condition can be ruled out. The comparison of the earlier data with the present results is summarized in Fig. 5 which represents the revised phase diagram for barium. Without the third dip in the melting curve of barium near $9 \mathrm{GPa}$ only the dip near $7.7 \mathrm{GPa}$ fits to the present results, where it is related on one side to the small stability range for the new $c F 4$ phase. The observation of this new high temperature phase is very surprising. No other alkaline 


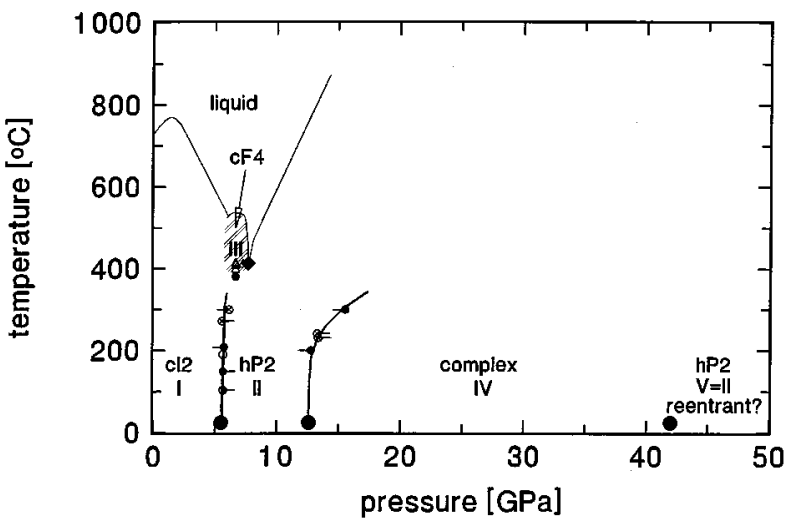

FIG. 5. Revised phase diagram for barium. The labeling of the data is the same as in Fig. 3.

earth metal shows this type of behavior. However, a $c F 4$ phase could be rationalized as a precursor for the next close packed $h P 2$ high pressure phase. Due to the lack of any recent evidence for the earlier spurious Ba- "III' phase, the assignment of the number III to this new phase can reestablish a systematic labeling in this phase diagram providing a liquid-I-III-triple point with respect to the earlier determination of the melting curve. ${ }^{29}$

Furthermore the data on the II-IV boundary from 12 to 17 $\mathrm{GPa}$ together with the temperature dependence of the $c / a$ ratio for $h P 2$ Ba-II lead to a significant modification of the phase diagram, Fig. 5, in comparison with the literature data presented in Fig. 3.

\section{CONCLUSION}

The unexpected observation of a $c F 4$ phase in barium along the melting curve around $5 \mathrm{GPa}$ together with the lack of any evidence for the spurious phase "III' previously assigned to the region from 8 to $12 \mathrm{GPa}$ at ambient temperature leads to the designation of $\mathrm{Ba}-\mathrm{III}$ to this new $c F 4$ phase. Together with the present first data for the slope of the II/IV boundary given in Fig. 5, one finds a drastic change in the topology of the phase diagram for barium along the melting curve. One can now speculate that the complex phase Ba-IV may represent only a "low temperature" structure which allows for a connection of the stability regions of both $h P 2$ structures $\mathrm{Ba}-\mathrm{II}$ and $\mathrm{Ba}-\mathrm{V}$ at high temperatures with the consequence that $\mathrm{Ba}-\mathrm{V}$ may just be reentrant $\mathrm{Ba}-\mathrm{II}$. This speculation needs however further experimental support.

\section{ACKNOWLEDGMENTS}

We would like to thank W. Sievers and W. Bröckling for technical assistance and J. Otto for taking care of the beam line. Financial support by the DFG under Contract No. Ho486/22-1 is gratefully acknowledged.
${ }^{1}$ S. Akimoto, T. Yagi, Y. Ida, and K. Inoue, High Temp. High Press. 7, 287 (1975).

${ }^{2}$ H. Olijnyk and W. B. Holzapfel, Phys. Lett. 100A, 191 (1984).

${ }^{3}$ H. Olijnyk and W. B. Holzapfel, High Pressure Geosciences and Material Synthesis (Academie Verlag, Berlin, 1988).

${ }^{4}$ K. Takemura, Phys. Rev. B 50, 16239 (1994).

${ }^{5}$ D. A. Young, Phase Diagrams of the Elements (University of California Press, Berkeley and Los Angeles, CA, 1991).

${ }^{6}$ J. C. Haygarth, I. C. Getting, and G. C. Kennedy, J. Appl. Phys. 38, 4557 (1967).

${ }^{7}$ J. P. Bastide, C. Susse, and R. Epain, C. R. Acad. Sci. 267, 857 (1968).

${ }^{8}$ J. P. Bastide and C. Susse, High Temp. High Press. 2, 237 (1970).

${ }^{9}$ M. A. Il'ina and E. S. Itskevich, JETP Lett. 11, 15 (1970).

${ }^{10} \mathrm{M}$. Winzenick and W. B. Holzapfel, in Proceedings of the Joint $X V$ AIRAPT and XXXIII EHPRG International Conference, Warsaw, 1995, edited by W. Trzeciakowski (Polish Acad. of Science, Warsaw, 1996), p. 384.

${ }^{11}$ J. C. Duthie and D. G. Pettifor, Phys. Rev. Lett. 38, 564 (1977).

${ }^{12}$ H. L. Skriver, Phys. Rev. B 31, 1909 (1985).

${ }^{13}$ J. A. Moriarty, Phys. Rev. B 34, 6738 (1986).

${ }^{14}$ K. Syassen and W. B. Holzapfel, Europhys. Conf. Abstr. 1A, 75 (1975).

${ }^{15}$ W. B. Holzapfel, in High Pressure Chemistry, edited by H. Kelm
(Reidel, Dordrecht/Holland, 1978).

${ }^{16}$ G. J. Piermarini, S. Block, and J. S. Barnett, J. Appl. Phys. 44, 5377 (1973).

${ }^{17}$ H. K. Mao, P. M. Bell, J. W. Shaner, and D. J. Steinberg, J. Appl. Phys. 49, 3276 (1978).

${ }^{18}$ W. A. Grosshans, E. F. Düsing, and W. B. Holzapfel, High Press. Res. 6, 169 (1991).

${ }^{19}$ J. Otto (unpublished)

${ }^{20}$ E. Grüneisen, Handb. Phys. 10, 1 (1926).

${ }^{21}$ V. N. Zharkov and V. A. Kalinin, Equations of State for Solids at High Pressures and Temperatures (Consultants Bureau, New York, London, 1971).

${ }^{22}$ M. Winzenick, Ph.D. thesis, Paderborn, 1996.

${ }^{23}$ S. N. Vaidya and G. C. Kennedy, J. Phys. Chem. Solids 31, 2329 (1970).

${ }^{24}$ M. S. Anderson, C. A. Swenson, and D. T. Peterson, Phys. Rev. B 41, 3329 (1990).

${ }^{25}$ F. Birch, Phys. Rev. 71, 809 (1947).

${ }^{26} \mathrm{~F}$. Porsch (unpublished).

${ }^{27}$ A. Yoneda and S. Endo, J. Appl. Phys. 51, 3216 (1980).

${ }^{28}$ S. T. Weir, K. Yogesh, K. Vohra, and A. L. Ruoff, Phys. Rev. B 33, 4221 (1986).

${ }^{29}$ A. Jayaraman, W. Klement, Jr., and G. C. Kennedy, Phys. Rev. Lett. 10, 387 (1963). 